\title{
Patrones de resistencia a antibióticos de Acinetobacter baumannii en un hospital de Colombia
}

Patterns of Acinetobacter baumannii resistance to antibiotics in a Colombian hospital

\author{
Mónica Chávez Ph.D1, Romel F. Gómez BSc², Cristina E. Cabrera MSc ${ }^{3}$, \\ Mario Esparza Ph.D ${ }^{4}$ \\ 'Profesor Titular. Facultad de Ciencias de la Salud. Universidad Libre, Cali, Colombia. Grupo de Investigación GIMMEIN. Profesor \\ Departamento de Ciencias Biomédicas. Universidad Santiago de Cali. Profesor, Departamento de Ciencias Biomédicas, Universidad \\ Santiago de Cali. \\ ${ }_{2}^{2}$ Profesor Auxiliar, Facultad de Ciencias de la Salud, Universidad Libre, Cali, Colombia. Grupo de Investigación GIMMEIN \\ ${ }^{3}$ Profesor Asociado, Facultad de Ciencias de la Salud, Universidad Libre, Cali, Colombia. Grupo de Investigación GIMMEIN. \\ Profesor Asociado, Facultad de Salud, Departamento de Microbiologia, Universidad del Valle. \\ ${ }^{4}$ Profesor Asistente. Facultad de Ciencias del Mar y Recursos Biológicos. Laboratorio de Biominería. Departamento de Biotecnología.
} Universidad Antofagasta, Chile.

\begin{abstract}
Resumen
Objetivo: Identificar patrones de resistencia en los aislamientos de $A$. baumannii obtenidos en una unidad de cuidados intensivos de Cali, Colombia. Diseño: Estudio descriptivo, prospectivo de corte transversal. Institución: Clínica Universitaria Rafael Uribe Uribe, Cali, Colombia. Materiales: Los aislamientos se obtuvieron de cultivos de muestras de sangre, heridas quirúrgicas, secreción nasal orina, secreción uretral y puntas de catéter. Intervenciones: Se recolectaron 52 aislamientos durante los años 2009 y 2010. Mediante el análisis del antibiograma se identificaron patrones de resistencia (antibiotipos), se realizó antibiograma cuantitativo y se construyó un cladograma basado en el agrupamiento por el método de promedios aritméticos de grupos apareados no ponderados (conocido en inglés como UPGMA). Principales medidas de resultados: Medida de la concentración mínima inhibitoria (CMI) y el coeficiente de similitud generado por las distancias de los diámetros de los halos de inhibición entre dos aislamientos (antibiograma cuantitativo). Resultados: Se identificaron 5 antibiotipos; el 50\% de los aislamientos se agruparon en el antibiotipo 1, con resistencia a todos los antibióticos y sensibilidad a tigeciclina y sulperazona; el antibiotipo 4 agrupó los aislamientos con resistencia a todos los antibióticos (19,3\%). En el antibiograma cuantitativo se identificaron dos clados con 5 y 47 aislamientos, respectivamente. Conclusiones: Los aislamientos de Acinetobacter baumannii tuvieron pocas diferencias fenotípicas y es probable que presenten alguna de las $\beta$-lactamasas tipo OXA.
\end{abstract}

Palabras clave: Acinetobacter baumannii, antibióticos, patrones de resistencia, antibiotipos, clados.

\section{Abstract}

Objective: To identify patterns of resistance in A. baumannii isolates obtained from an intensive care unit in Cali, Colombia. Design: Prospective cross-sectional descriptive study. Institution: Clínica Universitaria Rafael Uribe Uribe, Cali, Colombia. Materials: Isolates were obtained from cultures of blood, surgical wounds, nasal secretion, urine, urethral discharge and catheter tip samples. Interventions: Fifty-two isolates were collected between 2009 and 2010. Antibiogram analysis was done to identify resistance patterns (antibiotypes), a quantitative susceptibility testing was conducted, and a cladogram based on unweighted pair group method average (UPGMA) was constructed. Main outcome measures: Minimum inhibitory concentration (MIC) and similarity coefficient generated by diameters distances between two isolated inhibition zones (quantitative antibiogram). Results: Five antibiotypes were identified; $50 \%$ of isolates were grouped in antibiotype 1, were resistant to all antibiotics, and susceptible to tigecycline and sulperazone; antibiotype 4 grouped isolates resistant to all antibiotics (19.3\%). In the quantitative antibiogram two clades with 5 and 47 isolates were identified respectively. Conclusions: Acinetobacter baumannii isolates showed few phenotypic differences and probably present some of $\beta$-lactamases OXA type.

Keywords: Acinetobacter baumannii, antibiotics, susceptibility patterns, clusters.

\section{An Fac med. 2015;76(1):21-6 / doi:10.15381/anales.v76i1.11071}

\section{INTRODUCCIÓN}

Acinetobacter baumannii es un patógeno oportunista con una alta prevalencia en los hospitales de todo el mundo, especialmente en pacientes en estado crítico ${ }^{(1-3)}$. Posee la capacidad de adaptarse a varios ambientes y permanecer en superficies del ambiente hospitala- rio, como son pisos, lavaderos, manijas de las puertas y humidificadores, entre otros ${ }^{(4,5)}$. Esta característica, combinada con la resistencia simultánea que desarrolla a varios antibióticos contribuye a su capacidad de diseminación en los hospitales ${ }^{(2,3)}$.

En Colombia, los aislamientos de A. baumannii presentan la característica de multirresistencia ${ }^{(6-9)}$, especialmente a los antibióticos monobactámicos, inhibidores de $\beta$-lactamasas, cefalosporinas de tercera y cuarta generación, carbapenemes y aminoglicósidos. Estas cepas multirresistentes tienden a generar brotes epidémicos con el predominio de uno o dos cepas en más de 20 brotes ${ }^{(9)}$. 
Desde el punto de vista epidemiológico es importante determinar los mecanismos de transmisión de la bacteria y definir los patrones de transmisión en los brotes hospitalarios para elaborar estrategias de control, tomar conductas terapéuticas y de intervención para limitar el impacto clínico de estas infecciones. El abordaje precoz y efectivo de un brote, permite limitar el número de casos y prevenir su aparición en el futuro.

Una aproximación fenotípica que permite diferenciar varias cepas de una especie con genes que expresan mecanismos de resistencia es el análisis de los patrones de resistencia (antibiotipos) generados a partir de las pruebas de sensibilidad a los antibióticos. Otra aproximación fenotípica es el empleo del antibiograma cuantitativo; este se basa en las diferencias de sensibilidad entre los aislamientos de A. baumannii, para agruparlos a partir de las distancias de la CMI entre pares de aislamientos ${ }^{(10,11)}$.

Teniendo en cuenta la importancia de este microorganismo en las infecciones nosocomiales, el objetivo de este estudio fue identificar los patrones de resistencia de los aislamientos de $\mathrm{A}$. baumannii obtenidos de la UCI en un hospital de tercer nivel de la ciudad de Cali.

\section{MÉTODOS}

Este estudio fue desarrollado en la Clínica Universitaria Rafael Uribe Uribe, Institución de tercer nivel que cuenta con 475 camas, con una incidencia en los días de hospitalización aproximada de 6,9 por 1000 . Se diseñó un estudio descriptivo prospectivo de corte transversal, para identificar las características fenotípicas de los aislamientos de A. baumannii. Este estudio fue aprobado por el comité científico de ética y bioética de la Clínica Universitaria Rafael Uribe Uribe, Ciudad de Cali, Colombia.

Se recuperaron 52 aislamientos de A. baumannii en el periodo comprendido entre marzo de 2009 y julio de
2010. Los aislamientos se obtuvieron de muestras de sangre, herida quirúrgica, muestreo nasal, orina, secreción uretral y punta de catéter de pacientes internados en la UCI del hospital.

Las muestras fueron sembradas en agar Mc Conkey (Oxoid Ltd., Hampshire, United Kingdom) por 24 horas, a $37^{\circ} \mathrm{C}$, para el aislamiento y posterior identificación de la bacteria. La evaluación macroscópica de las colonias se realizó por observación de sus características morfológicas y los cambios producidos en el agar Mc Conkey, dentro de los que se encuentra la no fermentación de lactosa. El estudio microscópico se basó en el método de Gram para establecer morfología y tinción bacteriana. La identificación de los aislamientos pertenecientes al complejo A. calcoaceticus-A. baumannii se realizó en el laboratorio clínico del hospital, empleando el equipo Vitek GNI card (bioMeriexVitek Inc., Hazelwood, MO) ${ }^{(12)}$.

La prueba de sensibilidad a los antibióticos se desarrolló usando el método de difusión en disco en placas de agar Mueller Hinton (Scharlau Chemie S.A) con la calibración del inóculo basado en el estándar Mc-Farland, de acuerdo a los parámetros establecidos por el CLSI (Clinical and Laboratoty Standards Institute) ${ }^{(13)}$. Las cepas de A. baumannii ATCC 19606 y de Escherichia coli ATCC 25922 fueron empleadas como control de calidad.

Los antibióticos incluidos en la prueba de sensibilidad estaban contenidos en los sensidiscos (Oxoid) y correspondieron a: trimetroprim/sulfametoxazol (SXT 23,75 $\mu \mathrm{g}+1,25 \mu \mathrm{g}$ ), ticarcilina/ clavulanato (TIM $75 \mu \mathrm{g}-10 \mu \mathrm{g}$ ), gentamicina (GEN), tobramicina (TOB 10 $\mu \mathrm{g}$ ), ciprofloxacina (CIP $5 \mu \mathrm{g}$ ), ceftazidima (CAZ $30 \mu \mathrm{g}$ ), cefepima (FEP $30 \mu \mathrm{g}$ ), aztreonam (ATM), imipenem (IMP 10 $\mu \mathrm{g}$ ), meropenem (MEM $10 \mu \mathrm{g}$ ), ampicilina-sulbactam (SAM 10 $\mu \mathrm{g}-10 \mu \mathrm{g}$ ), amikacina (AMK $10 \mu \mathrm{g}$ ), cefoperazona/ sulbactam (sulperazona, SUL $75 \mu \mathrm{g}$ - 30 $\mu \mathrm{g})$ y tigeciclina (TIG $15 \mu \mathrm{g}$ ) (Oxoid). Los resultados del análisis fueron agrupados en patrones de resistencia.
Para el antibiograma cuantitativo, se partió de la comparación de los diámetros de los halos de inhibición en la técnica de difusión del disco (Kirby Bauer) (tabla 1). Se efectuó la sumatoria de los halos de inhibición realizando una comparación de un aislamiento de A. baumannii con otro, mediante fórmula matemática de la cual se desprende un coeficiente de similitud para los dos aislamientos bacterianos que se comparan. El coeficiente de similitud para calcular la distancia euclidiana se obtuvo por medio de la fórmula:

$$
E j k=\sqrt{ } \Sigma(x i j-x i k)^{2}
$$

donde xij y xik son los valores de los diámetros de inhibición de un antimicrobiano dado i para dos distintos aislamientos j y k. A un valor más bajo del coeficiente de similitud, mayor es la similitud entre dos cepas bacterianas (tabla 2). Se establecieron los valores del coeficiente de similitud para los 52 aislamientos, y de esta manera se construyó un cladograma basado en el agrupamiento por UPGMA.

La frecuencia de aislamientos con resistencia a los antibióticos se calculó como la prevalencia de pacientes infectados con A. baumannii resistente a los antibióticos. La diferencia en la frecuencia de las variables y su asociación con el tiempo de exposición intrahospitalario fueron determinadas por análisis de chi-cuadrado. Se calculó la sensibilidad, la especificidad y los valores predictivos positivos y negativos de los aislamientos con idéntica morfología de las colonias y el coeficiente de similitud del antibiograma cuantitativo $<10$, para determinar la correlación de cada prueba con la infección. Todos los valores de $\mathrm{p}$ fueron de dos colas; un valor de $p \leq 0,05$ se consideró estadísticamente significativo, empleando el paquete estadístico SPSS Vs20 (SPSS Inc, Chicago, IL).

\section{RESULTADOS}

En este estudio se obtuvo 24 aislamientos $(46,2 \%)$ a partir de muestreo nasal, 
Tabla 1. Valores de la concentración mínima inhibitoria (CMI) de cada antibiótico en los 52 aislamientos de $A$. baumannii obtenidos en la UCl de un hospital de Colombia. Los antibióticos evaluados fueron: SAM (ampicillina-sulbactam), CAZ (ceftazidima), CIP (ciprofloxacina), IMP (imipenem), LVX (levofloxacina), MEM (meropenem), TOB (tobramicina).

\begin{tabular}{cccccccc} 
Código & sam & caz & cip & imp & lev & mem & tob \\
5732549 & 16 & 2 & 8 & 2 & 8 & 64 & 8 \\
2513 & 16 & 2 & 8 & 4 & 8 & 64 & 8 \\
5759531 & 16 & 2 & 8 & 4 & 4 & 8 & 8 \\
5728549 & 16 & 16 & 4 & 8 & 4 & 8 & 8 \\
5717479 & 16 & 16 & 4 & 8 & 2 & 8 & 8 \\
5790341 & 16 & 16 & 2 & 8 & 4 & 8 & 8 \\
5793538 & 16 & 16 & 4 & 8 & 8 & 8 & 8 \\
\hline
\end{tabular}

12 aislamientos $(23,1 \%)$ de herida quirúrgica, $6(11,5 \%)$ de punta de catéter, $8(11,5 \%)$ de sangre y orina y 2 aislamientos $(3,8 \%)$ de secreción uretral.

El $100 \%$ de los aislamientos de A. baumannii presentó resistencia a amikacina, gentamicina, tobramicina, trimetoprim/sulfametoxazol, cefepima, ceftazidima, imipenem y ticarcilina/ clavulanato. Mientras que la resistencia a ciprofloxacina se detectó en $98,1 \%(51 / 52)$ de los aislamientos, a levofloxacina en 90,4\% (47/52), a ampicilina/sulbactam en $94,2 \%(49 / 52)$ y a meropenem en 96,2\% (50/52) (tabla 3). Los aislamientos presentaron menor resistencia a los antibióticos tigeciclina y sulperozona, con $21,2 \%$ y $28,8 \%$, respectivamente, siendo el mes de mayo del año 2010 la fecha que registró el mayor número de aislamientos con resistencia a sulperazona, con un valor significativo $(p<0,05)$.

Los aislamientos fueron considerados multirresistentes a los fármacos, basado en el criterio de resistencia simultánea a imipenem (o meropenem), amikacina (o tobramicina) y cefalosporinas de tercera generación (ceftazidima) ${ }^{(13)}$. El mayor número de estos aislamientos fue detectado en los meses de julio y septiembre del año 2009.

Diez aislamientos (19,3\%) fueron considerados pan-resistentes (PDR), basado en la resistencia simultánea a todos los antibióticos empleados en la prueba sensibilidad.
Con relación a un antibiotipo común en aislamientos co-circulantes, cinco patrones de resistencia (antibiotipos) fueron encontrados entre los 52 agrupamiento por UPGMA con los 52 aislamientos. (tigeciclina), LVX (levofloxacina). aislamientos de A. baumannii analizados (tabla 3). Los aislamientos considerados multirresistentes a fármacos fueron 26 y representaron el 50\%; estos aislamientos presentaron sensibilidad simultánea a tigeciclina y sulbactam y fueron clasificados en el antibiotipo 1; en este grupo se obtuvo quince (15) aislamientos de muestreo nasal y seis (6) de herida quirúrgica.

El grupo denominado antibiotipo 2 se presentó en nueve aislamientos $(17,3 \%)$ y se caracterizó por presentar multirresistencia a fármacos y sensibilidad a tigeciclina y sulperazona. Algunos de estos aislamientos presentaron sensibilidad también a levofloxacina, ampicilina/sulbactam o meropenem y fueron obtenidos de muestreo nasal (seis), punta de catéter (dos) y orina (uno).

Tabla 2. Distancia fenotípica entre los aislamientos de A. baumannii basados en el antibiograma cuantitativo. La distancia se calculó a partir del coeficiente de similitud comparando los diámetros de los halos de inhibición, donde xij - y xik son los valores de los diámetros de inhibición de un antimicrobiano dado i para dos distintos aislamientos j y $k$. A partir de estos valores se realizó un

\begin{tabular}{c|ccccccc} 
& 5732549 & 2513 & 5759531 & 5728549 & 5717479 & 5790341 & 5793538 \\
5732549 & 0 & 2 & 56 & 58 & 58 & 58 & 58 \\
2513 & & 0 & 56 & 56 & 58 & 58 & 58 \\
5759531 & & & 0 & 15 & 15 & 16 & 16 \\
5728549 & & & & 0 & 2 & 2 & 4 \\
5717479 & & & & & 0 & 3 & 6 \\
5790341 & & & & & & 0 & 16 \\
5793538 & & & & & & & \\
\hline
\end{tabular}

Tabla 3. Antibiotipos del 1 al 5, basado en el análisis del patrón de resistencia a los antibióticos, obtenido del antibiograma en los 52 aislamientos de A. baumannii. SXT (trimetroprim/ sulfametoxazol), TIM (ticarcilina/clavulanato), GEN (gentamicina), TOB (tobramicina), CIP (ciprofloxacina), CAZ (ceftazidima), FEP (cefepima), ATM (aztreonam), IPM (imipenem), MEM (meropenem), SAM (ampicillina-sulbactam), AMK (amikacina), SUL (cefoperazona-sulbactam), TIG

\begin{tabular}{|c|c|c|c|}
\hline Antibiotipo & $\begin{array}{l}\text { Número de } \\
\text { aislamientos }\end{array}$ & Resistencia & Sensibilidad \\
\hline 1 & 26 & $\begin{array}{l}\text { AMK, GEN, TOB, CIP, LVX, SXT, SAM, TIC/AC, } \\
\text { FEP, CAZ, IMP, MEM }\end{array}$ & TIG, SUL \\
\hline 2 & 9 & $\begin{array}{c}\text { AMK, GEN, TOB, CIP, SXT, TIC/AC, FEP, CAZ, } \\
\text { IMP }\end{array}$ & $\begin{array}{l}\text { TIG, SUL/ LVX, } \\
\text { SAM o MEM }\end{array}$ \\
\hline 3 & 7 & $\begin{array}{c}\text { AMK, GEN, TOB, CIP, LVX, SXT, SAM, TIC/AC, } \\
\text { FEP, CAZ, IMP, MEM }\end{array}$ & TIG o SUL \\
\hline 4 & 10 & $\begin{array}{c}\text { AMK, GEN, TOB, CIP, LVX, SXT, SAM, TIC/AC, } \\
\text { FEP, CAZ, IMP, MEM, TIG, SUL }\end{array}$ & - \\
\hline 5 & 1 & $\begin{array}{c}\text { AMK, GEN, TOB, SXT, SAM, TIC/AC, FEP, } \\
\text { CAZ, IMP, MEM }\end{array}$ & TIG, SUL, CIP, LVX \\
\hline
\end{tabular}


En el antibiotipo 3 se presentaron aislamientos multirresistentes a fármacos, pero sensibles a tigeciclina o sulperazona. Este antibiotipo se presentó en seis (6) aislamientos $(11,5 \%)$ obtenidos de sangre, orina, herida quirúrgica y muestreo nasal.

El antibiotipo 4 comprendió los diez aislamientos $(19,3 \%)$ pan-resistentes, con resistencia simultánea a todos los antibióticos evaluados, incluyendo tigeciclina y sulperazona, con una asociación estadísticamente significativa $(p<0,05)$. El antibiotipo 5 se detectó en un aislamiento $(1,9 \%)$ de sangre con susceptibilidad a tigeciclina, sulperazona y a las fluoroquinolonas (ciprofloxacina y levofloxacina).

El antibiotipo 1 se detectó durante el año 2009 en un aislamiento que circuló en el mes de junio, en julio (ocho), agosto (seis), septiembre (ocho), octubre (dos) y noviembre (uno). Solo un aislamiento con este antibiotipo se registró en el año 2010, en el mes de mayo. El antibiotipo 2 se obtuvo en aislamientos que circularon en el año 2009, en julio (dos), agosto (dos), septiembre (cuatro) y noviembre (uno). El antibiotipo 3 se detectó en dos aislamientos que circularon en los meses de septiembre y noviembre del año 2009 y en dos que circularon en mayo y uno en julio del año 2010. El antibiotipo 4 se detectó por primera vez en marzo del año 2009 y volvió aparecer en los meses de julio, agosto y noviembre del mismo año, y en los meses de abril, mayo y junio del año 2010. Finalmente, el antibiotipo 5 se detectó en el mes de octubre de 2009.

Aunque en el mes de septiembre de los años 2009 y 2010 se registró el mayor número de aislamientos, de los cuales 8 pertenecían al antibiotipo 1 y 4 al antibiotipo 2, no existió asociación estadísticamente significativa que relacionara la fecha en que circularon los aislamientos con un antibiotipo determinado $(p>0,05)$.

En el análisis cuantitativo del antibiograma, el cladograma basado en el agrupamiento por UPGMA a partir de las distancias de las CMI de los aislamientos evidenció la existencia de dos grupos. El clado 1 agrupó 5 aislamientos pertenecientes a los antibiotipos 2 , 3 y 4 , que circularon en septiembre de 2009 y en mayo de 2010. Estos aislamientos fueron obtenidos de muestras de orina, sangre, herida quirúrgica, punta de catéter y muestreo nasal. El clado 2 reunió todos los aislamientos pertenecientes al antibiotipo 1 y 5 , además de algunos aislamientos de los antibiotipos 2, 3 y 4 (figura 1).

\section{DISCUSIÓN}

Aunque las infecciones nosocomiales debidas a A. baumannii han sido encontradas en todo el mundo ${ }^{(1,2,5,6)}$, existe escasa información acerca del comportamiento epidemiológico de los aislamientos encontrados en la ciudad de Cali. La disponibilidad de 52 aislamientos nos proporcionó la oportunidad de examinar la sensibilidad a los antibióticos y las características fenotípicas.
En nuestro estudio, se aisló con más frecuencia el A. baumannii de muestras nasales de los pacientes internados en la UCI.

Los reportes del SENTRY en países de Latinoamérica informan que Acinetobacter presenta las mayores tasas de resistencia bacteriana y la resistencia a los carbapenemes es mayor que en Estados Unidos y Europa ${ }^{(14)}$. Así mismo, los estudios realizados por Picazo y col (3), en España, durante el año 2006, encontraron aislamientos con resistencia a la piperacilina/tazobactam en $90 \%$, a cefalosporinas de tercera generación en $70,4 \%$, a cefalosporinas de cuarta generación en $87,3 \%$. Un estudio similar para el año 2011, en el mismo país, registró 100\% de los aislamientos con resistencia a estos medicamentos ${ }^{(15)}$. En los Estados Unidos se reporta que más del 90\% de los aislamientos presenta resistencia a ciprofloxacina, y al menos $80 \%$ a cefalosporinas de espectro extendido, mientras que casi la mitad (49\%) es resistente a ampicilina/sulbactam. En nuestro estudio, los aisla-

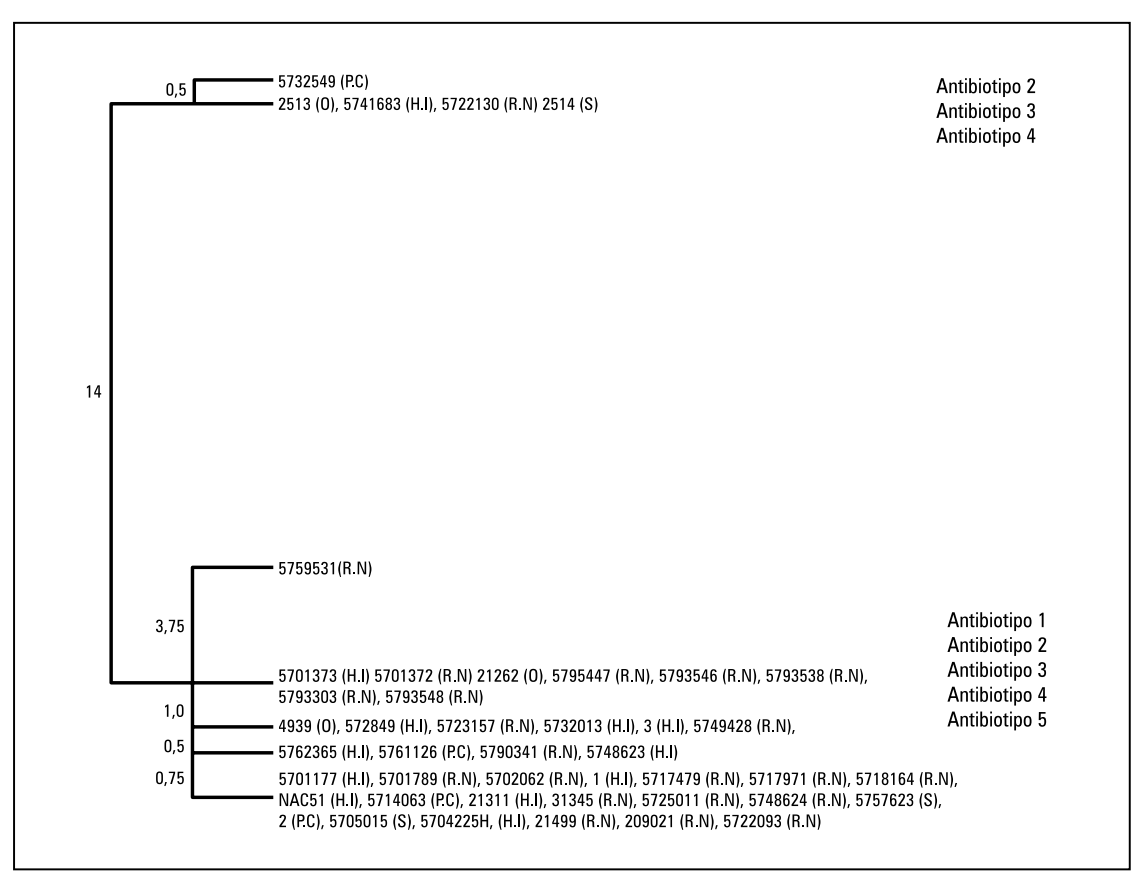

Figura 1. Cladograma basado en el agrupamiento por UPGMA del diámetro de inhibición de cada antimicrobiano para los 52 aislamientos de $A$. baumannii de un brote epidémico ocurrido en un hospital de alta complejidad de Colombia. Se formaron 2 grupos, el grupo 1 con 6 aislamientos con 3 antibiotipos en el grupo 2 con 46 aislamientos distribuidos en 5 antibiotipos. 
mientos obtenidos entre los años 2009 y 2010 fueron $100 \%$ resistentes a todos estos antibióticos ${ }^{(16)}$.

En el caso de la resistencia a los carbapenemes, un estudio multicéntrico realizado por Higgins y col (17), con aislamientos de A. baumannii en varios países durante 2010, mostró que el 100\% de ellos presentó resistencia a imipenem. En contraste, las comunicaciones de España informan que los aislamientos presentan una resistencia a imipenem de $60,8 \%$ y a meropenem de $57,6 \%$, para el año $2005^{(3)}$, y de $47,6 \%$ y $93,2 \%$ para el año $2011^{(15)}$. En los Estados Unidos, la resistencia a los carbapenemes se comunica alrededor de 20\% entre los años 2003 y $2005^{(16)}$. En nuestro estudio, la resistencia a los cabapenemes superó a los registros de España y Estados Unidos, con aislamientos resistentes a imipenem de $100 \%$ y a meropenem de $96,2 \%$, mostrando concordancia con los reportes del SENTRY.

En Colombia, un estudio realizado en UCI de 10 hospitales de tercer nivel comprobó que, de todas las bacterias estudiadas, A. baumannii presentaba los porcentajes más elevados de resistencia a antibióticos e incluso un aumento de resistencia a los carbapenemes ${ }^{(18)}$. En consonancia, los resultados del Grupo para el control de la Resistencia antimicrobiana de Bogotá (GREBO) resaltan un relativo incremento en la prevalencia de la resistencia al imipenem $(48,1 \%)$ y al meropenem (52\%) para el año $2006^{(19)}$ y Pinzón y col ${ }^{(7)}$ encuentran una resistencia a meropenem $(94,3 \%)$ e imipenem $(96,2 \%)$. Para el año 2012, Martínez y col registran una resistencia a imipenem y meropenem que se mantiene en $100 \%$ y $90 \%$, respectivamente ${ }^{(6)}$, lo que evidencia la estabilidad de los aislamientos de A. baumannii con resistencia a los carbapenemes en nuestro país.

Otro resultado importante en nuestro estudio fue la característica fenotípica de multirresistencia a fármacos en los aislamientos. Los brotes de cepas multirresistentes a fármacos han sido encontrados desde el año 2006 en los Estados Unidos, con 89\% de prevalencia ${ }^{(16)}$ y $100 \%$ de cepas multirresistentes en Asia ${ }^{(20,21)}$.

De acuerdo al concepto de pan-resistencia (22), en este estudio se detectaron cepas con esta característica en $19,3 \%$, con un valor estadísticamente significativo $(p<0,05)$. En un estudio adelantado en Brasil, en los años 2009 y 2010 se encontró aislamientos PDR en $11 \%{ }^{(23)}$.

Los agentes antibacterianos que aún son eficaces contra A. baumannii incluyen carbapenémicos, aminoglucósidos (gentamicina o amikacina), sulbactam, colistina y tigeciclina ${ }^{(24)}$. Sin embargo, en nuestro estudio los aislamientos de A. baumannii mostraron resistencia a todos estos agentes. Solo tigeciclina y sulperazona mostraron actividad antimicrobiana frente a la mayoría de los aislamientos evaluados (>80\%). Estos resultados son similares a los informes publicados para los aislamientos clínicos de otras regiones geográficas ${ }^{(22,25)}$. Karageorgopoulos y col ${ }^{(26)}$ consideran que la sensibilidad detectada a tigeciclina en por lo menos $90 \%$ de los aislamientos de Acinetobacter spp hace de este antibiótico una opción de tratamiento potencialmente eficaz contra las bacterias altamente resistentes. Aunque, hay que tener cautela con su uso, puesto que algunos informes indican que a pesar de la actividad de la tigeciclina in vitro, su eficacia se pierde significativamente in vivo, reportándose cada vez más casos de resistencia a este antibiótico ${ }^{(27,28)}$. Esto sugiere que la reciente introducción de la tigeciclina en la práctica clínica no constituye una solución definitiva al problema de la creciente resistencia a los medicamentos antimicrobianos en Acinetobacter spp., especialmente en A. baumannii.

En las tres combinaciones de $\beta$-lactámicos/inhibidoresde $\beta$-lactamasa evaluados, se observó que la combinación ampicilina-sulbactam y ticarcilina/ ácido clavulánico presentaron menor actividad frente a los aislamientos de A. baumannii; solo sulperazona presentó actividad antibacteriana significativa, lo que ya había sido comunicado ${ }^{(29-31)}$.

Boo y col ${ }^{(32)}$ plantean la posibilidad de la presencia de una co-selección de aislamientos resistentes a carbapenemes, mediados por la adquisición de carbapenemasas clase D tipo OXA. En nuestro estudio, todos los aislamientos presentaron capacidad para hidrolizar cefalosporinas de amplio espectro (ceftazidima y cefepima), y los carbapenemes como imipinem y meropenem, por lo que se sugiere la presencia de $\beta$-lactamasas de la clase D, tipo OXA23. Aunque esta oxacilinasa tiene baja eficiencia en la hidrólisis de los carbapenemes, la expresión simultánea de la OXA-51, así como la presencia de OXA-58, todas halladas en aislamientos de A. baumannii en Colombia y América Latina ${ }^{(17,33-34)}$, explicaría la resistencia tan marcada a los carbapenemes observada en nuestro estudio, como lo informaron Gales y col en su publicación ${ }^{(35)}$. Sin embargo, no se debe descartar que el alto nivel de resistencia en este tipo de oxacilinasas se presenta también asociado a otros mecanismos como la pérdida o reducción de expresión de proteínas de membrana y la sobre expresión de bombas de eflujo ${ }^{(36)}$.

En un estudio realizado por nuestro grupo en el mismo hospital durante los años 2007 y 2008, los aislamientos presentaron sensibilidad a las cefalosporinas de amplio espectro, como cefoperazona, ceftriazona, cefotaxime y cefepime y a los carbapenemes ${ }^{(8)}$. Sin embargo, se observó un incremento en la dinámica de la resistencia. Durante los años 2009 y 2010, los aislamientos presentaron resistencia a todos estos antibióticos, quedando como única opción terapéutica el empleo de tigeciclina y sulperazona. En este estudio predominaron aislamientos multirresistentes, distribuidos en cinco antibiotipos y agrupados en dos clústers, lo que demuestra una reducida diversidad fenotípica. Nuestros resultados apoyan los datos obtenidos por Villegas y col ${ }^{(9)}$, que demostraron que las cepas multirresistentes de A. baumannii tienden a 
generar brotes epidémicos con el predominio de uno a dos tipos de cepas.

El análisis fenotípico basado en las pruebas de sensibilidad a los antibióticos sigue siendo una herramienta importante para caracterizar las cepas de A. baumannii. La presencia de 5 antibiotipos y la agrupación en dos clados de los aislamientos con resistencia a múltiples fármacos, demuestra la capacidad de propagación de estos aislamientos, lo que contribuye a la aparición de brotes epidémicos.

\section{REFERENCIAS BIBLIOGRÁFICAS}

1. Peleg AY, Seifert H, Paterson DL. Acinetobacter baumannii: emergence of a successful pathogen. Clin Microbiol Rev. 2008 Jul;21(3):538-82. doi: 10.1128/CMR.00058-07.

2. Dijkshoorn L, Nemec A, Seifert $H$. An increasing threat in hospitals: multidrug-resistant Acinetobacter baumannii. Nat Rev Microbiol. 2007 Dec;5(12):939-51.

3. Picazo JJ, Betriu C, Rodriguez-Avial I, Culebras E, Gómez M, López F. Antimicrobial resistance surveillance: Vira Study 2006. Enferm Infecc Microbiol Clin. 2006;24(10):617-28.

4. Thom KA, Johnson JK, Lee MS, Harris AD. Environmental contamination because of multidrugresistant Acinetobacter baumannii surrounding colonized or infected patients. Am J Infect Control. 2011 Nov;39(9):711-5. doi: 10.1016/j. ajic.2010.09.005.

5. Hrenovica J, Durnb G, Goic-Barisicc I, Kovacicd A. Occurrence of an environmental Acinetobacter baumannii strain similar to a clinical isolate in paleosol from Croatia. Appl Environ Microbiol. 2014 May;80 (9):2860-6. doi: 10.1128/AEM.00312-14.

6. Martínez P, Mattar S. Imipenem-resistant Acinetobacter baumannii carrying the IsaBA1-BlaOXA-23, 51 and IsaB1-BlaaDC-7 genes in Monteria, Colombia. Braz J Microbiol. 2012 Oct:43(4):1274-80. doi: 10.1590/S1517-83822012000400006.

7. Pinzón JO, Mantilla JR, Valenzuela EM, Fernández F, Álvarez CA, Osorio E. Molecular characterization of Acinetobacter baumannii isolations from a burns unit in a third level attention hospital in Bogotá. Infect. 2006;10(2):71-8.

8. Chávez M, Salazar M, Cabrera C, Gómez R, Pallares $C$. Bacterias resistentes a los antibióticos en infecciones nosocomiales de un hospital en Colombia. Enf Inf Microbiol. 2012;33(4):19-25.

9. Villegas MV, Hartstein Al. Acinetobacter outbreaks, 1977-2000. Infect Control Hosp Epidemiol. 2003;24:284-95.

10. Labarca LJ. Utilización del antibiotipo como marcador epidemiológico en infecciones intrahospitalarias: Comparación con la epidemiologia molecular. Rev Chilena Infectol. 2002;19(Supl. 2):S157-60.

11. Bearson BL, Labarca JA, Brankovic LE, Cohen M, Bruckner DA, Pegues DA. Use of quantitative antibiogram analysis to determine the clonality of coagulase-negative Staphylococcus species from blood culture. Clin Microbiol Infect. 2004;10(2):148-55.
12. Bernards AT, Van der Toorn J, Van Boven CPA, Dijkshoorn L. Evaluation of the ability of the API20NE system to identify Acinetobacter genomic species. Eur J Clin Microbiol Infec Dis. 1996;15:303-8.

13. Clinical and Laboratory Standards Institute. Performance Standards for Antimicrobial Susceptibility Testing: Twenty-third Informational Supplement. 2013; M100-S23. CLSI, Wayne, PA, USA.

14. Gales AC, Castanheira M, Jones RN, Sader HS. Antimicrobial resistance among Gram-negative bacilli isolated from Latin America: results from SENTRY Antimicrobial Surveillance Program (Latin America, 2008-2010). Diagn Microbiol Infect Dis. 2012 Aug;73(4):354-60. doi: 10.1016/j. diagmicrobio.2012.04.007.

15. Villalón $P$, Valdezate $S$, Medina-Pascual MJ, Rubio VI, Vinde A, Saez JA. Clonal diversity of nosocomial epidemic Acinetobacter baumannii strains isolated in Spain. J Clin Microbiol. $2011 \mathrm{Mr}$;49(3):875-82. doi: 10.1128/JCM.01026-10

16. Hujer KM, Hujer AM, Hulten EA, Bajaksouzian S, Adams JM, Donskey C, et al. Analysis of antibiotic resistance genes in multidrug-resistant Acinetobacter $\mathrm{sp}$. isolates from military and civilian patients treated at the Walter Reed Army Medical Center of the isolates were resistant to at least three classes of antibiotics, hence meeting the criteria for multidrug resistance. Ant Agents Chem. 2006;50(12):4114-23.

17. Higgins PG, Dammhayn C, Hackel M, Seifert H. Global spread of carbapenem-resistant Acinetobacter baumannii. J Antimicrob Chemother. 2010 Feb;65(2):233-8. doi: 10.1093/jac/dkp428

18. Miranda MC, Pérez F, Zuluaga. Antimicrobial resistance in Gram- negative bacteria isolated from intensive care units of Colombian hospitals. WHONET 2003, 2004 and 2005. Biomédica. 2006;26:424-33.

19. Álvarez C, Cortés J. Resistencia antimicrobiana en unidades de cuidado intensivo de Bogotá, Colombia, 2001-2003. Rev Salud Pública. 2006;8(Supl.1):86-101.

20. Hong SB, Shin KS, Ha J, Han K. Co-existence of blaOXA-23 and armA in multidrug-resistant Acinetobacter baumannii isolated from a hospital in South Korea. J Med Microbiol. 2013 Jun;62(Pt 6):836-44. doi: 10.1099/jmm.0.055384-0.

21. Tan TY, Ng LS. Susceptibility of multi-resistant Gram-negative bacilli in Singapore to tigecycline as tested by agar dilution. Ann Acad Med Singapore. 2007 Oct;36(10):807-10.

22. Falagas ME, Kolets PK, Bliziotis IA. The diversity of definitions of multidrug-resistant (multirresistencia a fármacos) and pandrug-resistant (PDR) Acinetobacter baumannii and Pseudomonas aeruginosa. J Med Microbiol. 2006;55:1619-29.

23. Prata-Rocha ML, Gontijo-Filho PP, Batista de Melo G. Factors influencing survival in patients with multidrug resistant Acinetobacter baumannii infection. Braz J Infect Dis. 2012 May-Jun;16(3):237-41.

24. Rafailidis PI, loannidou EN, Falagas ME. Ampicillin/ sulbactam: current status in severe bacterial infections. Drugs. 2007;67(13):1829-49.

25. Waites KB, Duffy LB, Dowzicky MJ. Antimicrobial susceptibility among pathogens collected from hospitalized patients in the United States and in vitro activity of tigecycline, a new glycylcycline antimicrobial. Antimicrob Agents Chemother. 2006;50:3479-84.

26. Karageorgopoulos DE, Kelesidis T, Kelesidis I, Falagas ME. Tigecycline for the treatment of multidrug-resistant (including carbapenemresistant) Acinetobacter infections: a review of the scientific evidence. J Antimicrob Chemother. 2008 Jul;62(1):45-55. doi: 10.1093/jac/dkn165.

27. Navon-Venezia, S, Leavitt A, Carmeli Y. High tigecycline resistance in multidrug-resistant Acinetobacter baumannii. J Antimicrob Chemother. 2007 Apr;59(4):772-4.

28. Reid GE, Grim SA, Aldeza CA, Janda WM, Clark NM. Rapid development of Acinetobacter baumannii resistance to tigecycline. Pharmacotherapy. 2007 Aug;27(8):1198-201.

29. Gales AC, Jones RN, Sader HS. Global assessment of the antimicrobial activity of polymyxin B against 54731 clinical isolates of Gram-negative bacilli: report from the SENTRY antimicrobial surveillance programme (2001-2004). Clin Microbiol Infect. 2006;12:315-21.

30. Song JY, Kee SY, Hwang IS. In vitro activities of carbapenem/sulbactam combination, colistin, colistin/ rifampicin combination and tigecycline against carbapenem-resistant Acinetobacter baumannii. J Antimicrob Chemother. 2007;60:317-22.

31. Tong W, Wang R, Chai D, Li Z, Pei F. In vitro activity of cefepime combined with sulbactam against clinical isolates of carbapenem resistant Acinetobacter spp. Int J Antimicrob Agents. 2006;28:454-6.

32. Boo TW, Walsh F, Crowley B. Molecular characterization of carbapenem-resistant Acinetobacter species in an Irish university hospital: predominance of Acinetobacter genomic species. J Med Microbiol. 2009 Feb;58(Pt 2):209-16. doi: 10.1099/ jmm.0.004911-0.

33. Villegas M, Kattan N, Correa A, Lolans K, Guzman A, Woodford N, Livermore D, Quinn J, and the Colombian Nosocomial Bacterial Resistance Study Group (2007) Dissemination of Acinetobacter baumannii clones with OXA-23 carbapenemase in Colombian hospitals. Antimicrob Agents Chemother. 2007 Jun;51(6):2001-4.

34. Opazo A, Domínguez M, Bello H, Amyes S, González G. OXA-type carbapenemases in Acinetobacter baumannii in South America. J Infect Dev Ctries. 2012 Apr 13;6(4):311-6.

35. Gales AC, Castanheira M, Jones RN, Sader HS. Antimicrobial resistance among Gram- negative bacilli isolated from Latin America: results from SENTRY Antimicrobial Surveillance program (Latin America, 2008-2010). Diagn Microbiol Infect Dis. 2012 Aug;73(4):354-60. doi: 10.1016/j. diagmicrobio.2012.04.007.

36. Poirel L, Nordmann P. Carbapenem resistance in Acinetobacter baumannii: mechanisms and epidemiology. Clin Microbiol Infect. 2006;12:826-36.

Artículo recibido el 1 de agosto de 2014 y aceptado para publicación el 11 de noviembre de 2014.

Conflictos de interés: Los autores declaran no existir conflictos de interés.

\section{Correspondencia:}

Mónica Chavez, Ph.D.

Facultad de Ciencias de la Salud, Universidad Libre, Cali, Colombia

Dirección: Diagonal 37A No3-29. Barrio Santa Isabel

Teléfono: (57-2) 5240007, ext 1337

Correo electrónico: monikchavez@gmail.com 